\title{
Cancers associés aux herpèsvirus
}

\section{Diversité clinique, similitudes biologiques}

Pierre Busson, Catherine Pioche-Durieu, Vincent Maréchal, Irène Joab

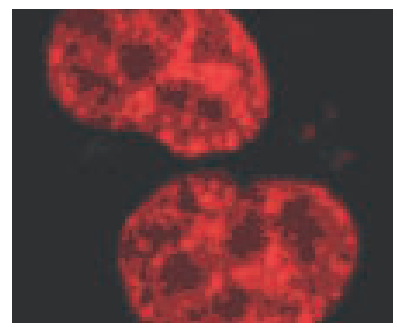

Les affections tumorales associées aux herpèsvirus existent dans toutes les grandes classes de vertébrés et produisent chez l'homme des tableaux cliniques très variés, du sarcome de Kaposi à la maladie de Hodgkin en passant par les carcinomes nasopharyngés. De prime abord, l'investigation biologique de ces maladies est d'autant plus difficile qu'elles ont toujours une étiologie multifactorielle, le virus étant un facteur pathogène nécessaire mais non suffisant. Néanmoins, une approche multidisciplinaire et comparative révèle des mécanismes physiopathologiques généraux communs à plusieurs catégories nosologiques. C'est l'un des principaux enseignements du colloque «Herpesviruses and cancer. Advances in basic biology and clinical research » qui, en mars 2007, a analysé un ensemble de pathologies tumorales liées à EBV (Epstein-Barr), HHV8 (human herpesvirus 8, virus du sarcome de Kaposi), HHV6, au cytomégalovirus et au virus de la maladie de Marek ${ }^{1}$.

\section{Facteurs de risques et événements pré-tumoraux}

En règle générale, les tumeurs associées aux herpèsvirus n'affectent qu'un petit nombre d'individus par rapport à l'ensemble des sujets infectés dans une espèce donnée. D'où l'importance d'une bonne compréhension des facteurs de risque en rapport avec l'hérédité, les modalités de primo-infection, le fonctionnement de la réponse immunitaire ou encore l'existence de souches virales plus agressives. L'exposé de S. Plancoulaine (Nec-

${ }^{1}$ Virose aviaire extrêmement contagieuse transmise par un herpèsvirus. ker, Paris, France) a fait le point sur les facteurs génétiques de susceptibilité aux infections par les herpèsvirus. Dans certains cas rares, la vulnérabilité à l'infection virale est liée à une altération monogénique $[1,2]$. Plus souvent, la susceptibilité est modulée par plusieurs gènes. C'est le cas par exemple dans la maladie de Hodgkin associée au virus d'Epstein-Barr. Une étude d'association présentée par C. Besson (Kremlin-Bicêtre, France) a mis en évidence une modulation du risque en fonction du polymorphisme des gènes allèles codant les récepteurs Kir activateurs des cellules NK (certains allèles augmentant le risque, d'autres étant protecteurs).

De façon remarquable, la communication de D. Sauce (Birmingham, Royaume-Uni) sur la « cicatrice immunologique » de la mononucléose infectieuse a mis en exergue un autre type de facteur de risque lié aux modalités de la primo-infection virale. On sait que l'infection initiale par EBV est presque toujours silencieuse chez l'enfant alors qu'elle s'accompagne très souvent d'une mononucléose infectieuse (MNI) chez l'adolescent ou le jeune adulte. En outre, des antécédents de MNI augmentent le risque de maladie de Hodgkin associée à EBV [3]. Les travaux de D. Sauce montrent que la MNI entraîne une extinction durable - et peut-être même 
définitive - de l'expression du récepteur $\alpha$ de l'IL-15 (IL-15R $\alpha$ ) par les lymphocytes T et NK, ce qui est susceptible de retentir de façon défavorable sur la surveillance immunitaire anti-virale et anti-tumorale [4]. L'étude se poursuit en ce moment même pour savoir si un déficit similaire peut s'observer chez les patients atteints de maladie de Hodgkin notamment quand celle-ci est associée à l'EBV.

Le risque d'aboutir à une pathologie tumorale peut également être influencé par la rencontre de souches virales ayant un pouvoir oncogénique plus fort. Ce point a été bien illustré par l'exposé d'A. Chanut (groupe de J. Feuillard, Limoges, France) montrant qu'on peut distinguer des isoformes plus ou moins oncogéniques de la LMPI (latent membrane protein 1) d'EBV sur la base d'un test de transformation de lymphocytes murins $B a / F 3$. Les isoformes en provenance d'un donneur sain ne sont oncogéniques qu'une fois sur deux alors qu'elles le sont toujours quand elles proviennent de cellules de Hodgkin.

\section{Mécanismes d'oncogenèse et de croissance tumorale}

Dans la plupart des affections tumorales associées à des herpèsvirus, le génome viral est contenu dans la totalité des cellules malignes. Selon la nature de la tumeur et du virus, un petit nombre de gènes viraux sont exprimés de façon régulière et spécifique. Les produits de ces gènes viraux altèrent de nombreux mécanismes de régulation en relation avec la survie et la prolifération cellulaires, la réponse aux interférons, les mécanismes de réparation de l'ADN etc. La compréhension de ces altérations est une entreprise de longue haleine mais elle est essentielle pour alimenter en amont les études de prédisposition génétique (gènes candidats) et en aval l'exploration de nouvelles approches thérapeutiques. À titre d'exemple, citons l'effet antagoniste de la LMP2 d'EBV sur les mécanismes de réponse aux interférons $\alpha$ et $\gamma$ (L. Young, Birmingham), la surexpression de la polymérase $\beta$ induite par la LMPI d'EBV, facteur d'instabilité génétique (groupe de F. Megetto, Toulouse, France), la surexpression de STATl également induite par la LMPI (groupe de R. Fagard, Bobigny, France), l'inactivation de la PP2A par une isoforme courte d'EBNA-LP

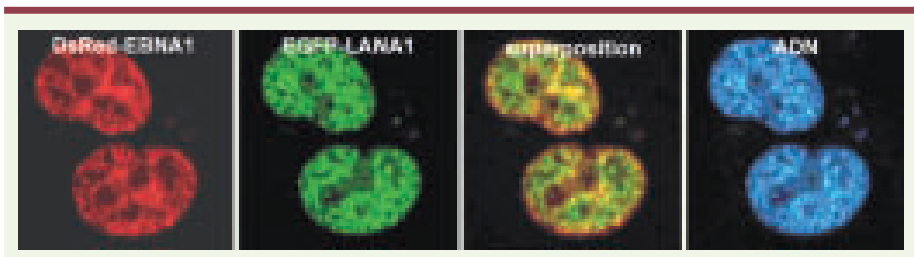

Figure 1. Cellules transfectées co-exprimant artificiellement les protéines EBNAI (en rouge) et LANAI (en vert). EBNAl et LANAl sont deux protéines de localisation nucléaire, codées respectivement par EBV et par HHV8 et exprimées en situation d'infection latente. Bien qu'elles ne présentent pas d'homologie de séquence, ces deux protéines présentent de nombreuses analogies fonctionnelles: interaction avec la chromatine en interphase (photo) et en mitose, recrutement des génomes viraux sur les chromosomes mitotiques, activation de la réplication et contrôle de la ségrégation des épisomes viraux (source: V. Maréchal, non publiée).
d'EBV (groupe de J. Wiels, IGR, Villejuif, France). La liste des produits oncogéniques des herpèsvirus ne se limite pas aux protéines. Certains ARN non traduits jouent sans doute un rôle déterminant eux aussi. Par exemple, le virus de la maladie de Marek code une sous-unité ARN du complexe télomérase capable de coopérer avec la sous-unité catalytique codée par la cellule hôte. Ensemble, les deux sous-unités pourraient contribuer à la transformation des leucocytes de poulet $[5,6]$. Comme l'a rappelé S. Pfeffer (Strasbourg, France), la production de micro-ARN est une des caractéristiques des herpèsvirus, notamment $E B V$ et HHV8 [7]. Ces miARN semblent avoir pour fonction principale de bloquer l'expression de certains gènes cellulaires. L'une des approches utilisées pour l'identification des gènes cibles fait appel à des analyses comparatives du profil transcriptionnel sur biopuces.

Il est utile de pouvoir confirmer l'activité oncogénique des produits viraux par des observations réalisées directement sur des échantillons cliniques comme l'a fait le groupe de R. Küppers (Essen, Allemagne) dans ses travaux sur EBV et maladie de Hodgkin. En montrant notamment que les cellules de Reed-Sternberg sont infectées par $\varepsilon B V$ dans $100 \%$ des cas lorsque leurs gènes d'immunoglobulines sont affectés par une mutation non-sens (contre environ $40 \%$ dans le cas contraire). Cela tend à prouver que les signaux anti-apoptotiques $d^{\prime} \ell B V$ se substituent à ceux qui sont transmis normalement par le récepteur des cellules $B$ dans les centres germinatifs, entraînant une survie aberrante des cellules $B$ en voie de transformation [8].

S'il est certain que les produits des herpèsvirus - protéines ou ARN - modifient profondément le fonctionnement cellulaire, la réciproque est également vraie. Il est essentiel de ne jamais oublier que l'activité transformante des herpèsvirus dépend très étroitement du contexte cellulaire. Celui-ci influe sur l'activité des protéines associées à la latence virale et au cycle lytique. Par exemple EBNAl (EBV) coopère avec les protéines chromatiniennes HMGBl et 2 pour moduler l'expression de promoteurs placés sous son contrôle et, potentiellement, pour contrôler l'activité de l'origine de réplication durant la latence (groupe de V. Maréchal, Paris). L'activité du principal transactivateur du cycle lytique d'EBV, ZEBRA, peut être modulée par des cytokines comme le TGF- $\beta 1$ (groupe d'I. Joab, IAL, Villejuif) et par des protéines intracellulaires comme l'ubinucléine (H. Gruffat, Lyon, France).

En dehors des modèles de laboratoire, les virus oncogènes suffisent rarement, seuls, à la mise en place et à l'entretien du phénotype transformé. Comme nous l'avons souligné en introduction, les affections mali- 
gnes associées aux herpèsvirus ont toujours un caractère multifactoriel. Ainsi chez les sujets immunodéprimés, le développement de certains lymphomes résulte à la fois de l'infection par EBV et d'une altération des mécanismes de réparation des mésappariemments entre bases (J. Duval, hôpital Saint-Louis, Paris) [9]. Dans les carcinomes nasopharyngés associés à $\varepsilon B V$, on constate une surexpression récurrente de la protéine anti-apoptotique c-IAP2 qui paraît s'ajouter à l'infection par EBV (groupe de P. Busson, Villejuif).

\section{L'émergence du concept d'oncomodulation}

Dans le modèle le plus classique d'association entre tumeur et herpèsvirus, le génome viral est présent sous forme monoclonale dans la totalité des cellules du clone malin. En réalité, d'autres modalités d'association peuvent exister, par exemple dans le sarcome de Kaposi où le génome d'HHV8 peut avoir une distribution polyclonale (A. Gessain, Institut Pasteur, Paris). Mais il y a plus, dans certaines pathologies tumorales, on suspecte le rôle étiologique de tel ou tel herpèsvirus sans qu'il y ait persistance du génome dans la majorité des cellules malignes. Il s'agit d'une question controversée mais potentiellement d'une très grande importance pour l'ensemble de la cancérologie humaine. L'exposé de C. Davrinche a porté sur la contribution possible du CMV au développement des neuroblastomes. L'ADN du CMV est souvent détecté par PCR dans ces tumeurs pédiatriques. Or, l'infection de cellules de neuroblastome en culture par le CMV stabilise la protéine deltaN p73, une forme dominantenégative des produits des anti-oncogènes $p 73$ et $p 53$. II en résulte une plus grande résistance à l'apoptose [10]. Ces observations ont conduit les auteurs à proposer l'hypothèse d'une contribution du CMV à la progression tumorale in vivo; le CMV permettrait à un petit nombre de cellules malignes de franchir une étape supplémentaire dans la mise en place d'un phénotype plus agressif. D'autres protéines cellulaires comme Cox2 pourraient participer à d'autres effets oncomodulateurs du CMV. Le même concept pourrait s'appliquer à EBV dont on suspecte le rôle dans la progression de certains cancers épithéliaux (poster du groupe d'I. Joab, IAL, VillejuiF [11].

\section{De nouvelles armes thérapeutiques}

Les aspects thérapeutiques n'ont pas été oubliés. L'étude structurale des oncoprotéines virales est tout à fait essentielle pour la conception de petites molécules inhibitrices capables de diffuser dans les tissus et les compartiments cellulaires concernés (communication du groupe de W. Burmeister, Grenoble, France). Deux communications ont porté sur des approches de vaccination thérapeutique ciblant des protéines d'EBV. L'une est fondée sur l'utilisation de peptides présentés par un large éventail de molécules HLA de classe II stimulant l'expansion in vitro de lymphocytes CD4 spécifiques des protéines EBNAl, LMPl et 2 d'EBV (peptides promiscuous dessinés à l'aide du logiciel TEPITOPE) (0. Morales, IBL, Lille, France) [12]. L'autre consiste à déclencher la production d'anticorps dirigés contre des épitopes conformationnels portés par les boucles externes de protéines virales membranaires de type III, comme les LMPl et 2 (poster du groupe de P. Busson et D. Tranchand-Bunel, IGR, Villejuif). Enfin des expériences réalisées in vitro et sur des tumeurs xénogreffées indiquent que le parvovirus $\mathrm{Hl}$ apparaît comme un agent oncolytique prometteur dans certaines lignées de lymphome de Burkitt infectées de façon latente par EBV (poster du groupe de J. Rommelaere, DKFZ, Heidelberg, Allemagne). $\diamond$

Cancers associated with herpesviruses:

clinical diversity, biological similarities

\section{REMERCIEMENTS}

Le colloque «Herpesviruses and cancer. Advances in basic biology and clinical research » a été organisé le 2 mars 2007 au Centre de Recherche des Cordeliers, à Paris, par le réseau «Herpèsvirus et Cancer», avec le soutien de l'Inserm.

\section{RéFÉRENCES}

1. Rigaud S, Fondaneche MC, Lambert N, et al. XIAP deficiency in humans causes an X-linked lymphoproliferative syndrome. Nature $2006 ; 444: 110-4$.

2. Casrouge $A$, Zhang SY, Eidenschenk $C$, et al. Herpes simplex virus encephalitis in human UNC93B deficiency. Science $2006 ; 314: 308-12$.

3. Hjalgrim H, Askling J, Rostgaard K, et al. Characteristics of Hodgkin's lymphoma after infectious mononucleosis. N Engl J Med 2003 ; 349 : 1324-32.

4. Sauce D, Larsen M, Curnow SJ, et al. EBV-associated mononucleosis leads to long-term global deficit in T-cell responsiveness to IL-15. Blood 2006; 108 : 11-8.

5. Fragnet L, Blasco MA, Klapper W, Rasschaert D. The RNA subunit of telomerase is encoded by Marek's disease virus. J Virol $2003 ; 77: 5985-96$.

6. Shkreli M, Dambrine G, Soubieux D, et al. Involvement of the oncoprotein C-Myc in viral telomerase RNA gene regulation during Marek's disease virus-induced lymphomagenesis. J Virol $2007 ; 81: 4848-57$.

7. Pfeffer $S$, Zavolan M, Grasser FA, et al. Identification of virus-encoded microRNAs. Science $2004 ; 304: 734-6$.

8. Brauninger A, Schmitz R, Bechtel D, et al. Molecular biology of Hodgkin's and Reed/Sternberg cells in Hodgkin's lymphoma. Int J Cancer 2006 ; 118 : 1853-61.

9. Duval A, Raphael M, Brennetot $C$, et al. The mutator pathway is a feature of immunodeficiency-related lymphomas. Proc Natl Acad Sci USA 2004 ; 101 : 5002 -7.

10. Terrasson J, Allart S, Martin H, et al. p73-dependent apoptosis through death receptor: impairment by human cytomegalovirus infection. Cancer Res $2005 ; 65: 2787-94$.

11. Arbach $H$, Viglasky V, Lefeu F, et al. Epstein-Barr virus ( $(B B V)$ genome and expression in breast cancer tissue: effect of $\varepsilon B V$ infection of breast cancer cells on resistance to paclitaxel (Taxol). J Virol $2006 ; 80: 845-53$.

12. Depil S, Morales 0 , Castelli FA, et al. Determination of a HLA II promiscuous peptide cocktail as potential vaccine against EBV latency II malignancies. J Immunother 2007 ; $30: 215-26$.

\section{TIRÉS À PART}

P. Busson

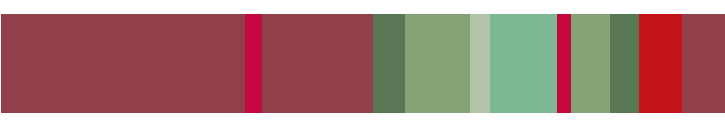

